\title{
How can naturally occurring fatty acids neutralize Listeria?
}

\author{
Birgitte H Kallipolitis*,1 \\ ${ }^{1}$ Department of Biochemistry \& Molecular Biology, University of Southern Denmark, Campusvej 55, 5230 Odense M, Denmark \\ * Author for correspondence: bhk@bmb.sdu.dk
}

\section{"Most recently, alternative options to the traditional antibiotics have been proposed, such as inhibitory compounds reducing the virulence of Listeria"}

First draft submitted: 14 August 2017; Accepted for publication: 15 August 2017; Published online: 4 October 2017

Keywords: antivirulence $\bullet$ infection $\bullet$ Listeria

Listeria monocytogenes is a Gram-positive bacterial pathogen causing severe foodborne disease listeriosis [1]. It thrives in a broad range of environments and grows under conditions traditionally used for food preservation, such as low temperature, low $\mathrm{pH}$ and high salt concentrations. After ingestion of contaminated food products, the bacterium enters the GI tract. In at-risk populations (pregnant individuals and elderly or otherwise immunocompromised individuals), Listeria may cross multiple protective barriers within the body, causing severe complications such as spontaneous abortion, septicemia or meningitis [1]. The high lethality rates of infected individuals (up to 30\%), even with antibiotic treatment, clearly demonstrate that listeriosis represents a severe clinical challenge. Most recently, alternative options to the traditional antibiotics have been proposed, such as inhibitory compounds reducing the virulence of Listeria [2-4]. One of these options relies on the observation that naturally occurring medium- and long-chain free fatty acids (FFAs) efficiently prevent Listeria from expressing its key virulence factors [4]. In this editorial, recent examples of antivirulence agents acting on Listeria will be discussed.

\section{The intracellular lifestyle of Listeria}

During infection, L. monocytogenes gains access to the cytoplasm of a variety of host cells [1]. The bacterium multiplies intracellularly and spreads to neighboring cells through host actin polymerization. Key virulence factors mediating invasion and intracellular replication by L. monocytogenes are well-characterized [1]. Specific surface proteins (e.g., InlA and InlB) are essential for bacterial entry into nonphagocytic cells, whereas listeriolysin $\mathrm{O}$ (LLO) and phospholipases (PlcA and $\mathrm{PlcB}$ ) mediate the escape from the host cell phagosome. Once inside the host cell cytosol, the surface protein ActA promotes actin polymerization and cell-to-cell movement. Expression of these and other virulence factors in Listeria is regulated by PrfA, a transcriptional activator protein belonging to the Crp/Fnr family of regulators [1,5]. To activate transcription, PrfA binds as a homodimer to a specific DNA sequence, the PrfA box, found in the promoter region of PrfA-regulated genes. When Listeria resides in the external environment, PrfA most often takes up an 'inactive' confirmation, which binds to the PrfA box albeit with low affinity $[1,5]$. In the intracellular environment, the activity of PrfA increases dramatically, mainly due to the binding of bacterial- and host-derived glutathione [6]. Upon binding of this cofactor, PrfA undergoes a conformational change in its 'active' form, holding an optimal conformation for PrfA-DNA interaction [7]. Mutant variants of PrfA, called PrfA*, are known to lock the protein in its active conformation [1,5,7]. When Listeria carries such a $p r f A^{*}$ mutation, PrfA-regulated virulence genes are constitutively expressed irrespective of the growth conditions. Importantly, a mutant Listeria lacking $\operatorname{prfA}$ (e.g., $\triangle \operatorname{prf} A$ ) is avirulent.

\section{Antivirulence strategies against Listeria?}

Due to the rapid increase in antibiotic resistance, alternatives to classical antibiotics are receiving increasing attention, such as drugs that intervene with bacterial virulence [8,9]. Antivirulence drugs act by disarming the pathogen of its virulence factors and may be used either alone or together with antibiotics. In the case of Listeria, 
PrfA represents an obvious target for antivirulence compounds. Indeed, several recent studies focused on the identification and characterization of compounds acting to prevent PrfA-dependent virulence gene expression. First, a study demonstrated that ring-fused 2-pyridone molecules attenuate the virulence of Listeria by reducing the expression of PrfA-activated virulence genes [2]. More specifically, these drugs bind directly to PrfA to reduce its DNA binding activity. Importantly, a PrfA* mutant was not affected, suggesting that ring-fused 2-pyridones could act to lock PrfA wt in its inactive form. Indeed, structural characterization of the interaction between PrfA and a ring-fused 2-pyridone confirmed that the drug binds to regions important for PrfA activation and DNA binding [2]. A purine analog (6- $N$-hydroxylaminopurine [6- $N$-HAP]) represents another recent example of an antivirulence drug acting on Listeria [3]. 6-N-HAP was shown to decrease virulence gene expression in Listeria by reducing the level as well as the activity of PrfA wt. In addition to its antivirulence effect, 6- $N$-HAP also acts as a potent mutagen in Listeria, thereby reducing its viability. At last, naturally occurring medium- and long-chain FFAs, such as the omega-3 fatty acid eicosapentaenoic acid (C20:5), were shown to prevent PrfA-dependent activation of virulence genes in Listeria [4]. As a foodborne pathogen, Listeria encounters a variety of FFAs in foods and in the GI tract. Some naturally occurring FFAs exhibit potent antimicrobial activity against Listeria, but the exact mechanism underlying their antimicrobial effect is not well understood. Additionally, specific medium- and long-chain FFAs have antivirulence effects on Listeria. First, the invasive efficiency of Listeria in enterocyte-like cells is strongly decreased in the presence of subinhibitory concentrations of medium- and long-chain FFAs naturally present in milk [10]. Second, the expression of LLO, ActA and other PrfA-dependent virulence factors is strongly downregulated upon exposure to subinhibitory concentrations of specific medium- and long-chain FFAs, including eicosapentaenoic acid [4]. Thus, some FFAs not only exhibit antibacterial activity against Listeria; they also act to prevent Listeria from expressing its key virulence factors.

\section{How do naturally occurring free fatty acids sabotage Listeria virulence?}

The PrfA regulator is likely to be the target of the medium- and long-chain FFAs acting by an antivirulence mechanism. Notably, the inhibitory effect of FFAs on virulence gene expression is also observed in Listeria encoding the constitutively active mutant variant PrfA* [4]. After $1 \mathrm{~h}$ of FFA exposure, transcription of PrfAdependent virulence genes is strongly reduced, but the level of $\operatorname{PrfA}^{*}$ protein remains unaffected at this time point. These findings suggest that FFAs act by blocking the activity of PrfA, possibly by generating an inhibitory signaling event via the membrane, or by direct binding to the PrfA protein itself [4]. Interestingly, unsaturated long-chain FFAs present in bile are known to inhibit the expression of two primary virulence genes in Vibrio cholera, encoding the cholera toxin and toxin-coregulated pilus [11]. The transcription factor ToxT, belonging to the AraC family, directly activates expression of cholera toxin and toxin-coregulated pilus, but the presence of unsaturated long-chain FFAs inhibits ToxT-dependent activation. Structural and functional analyses of ToxT revealed that unsaturated long-chain FFAs bind directly to a regulatory region in ToxT, suggesting that they prevent ToxT dimerization and/or DNA binding [12-14]. Importantly, unsaturated long-chain FFAs have been shown to also inhibit the activity of another member of the AraC family, the HilD virulence regulator in Salmonella enterica [15]. Structural and functional studies on PrfA in Listeria could show if FFAs also bind directly to Crp/Fnr family members. If so, structural analyses of the binding of FFA to PrfA might reveal the mechanism underlying the inhibitory effect of FFAs in Listeria and could form the basis for the future design of antivirulence compounds against Listeria and potentially other pathogens encoding virulence regulators of the Crp/Fnr family. Indeed, the x-ray structure of the unsaturated FFA palmitoleic acid (C16:1) bound to ToxT has served as a template for the design of a new class of highly effective ToxT inhibitors [16]. These small-molecule inhibitors were designed to resemble the folded fatty acid and bind very tightly to ToxT. Importantly, the compounds inhibit virulence gene expression even more efficiently than unsaturated FFAs and inhibit ToxT-DNA binding interaction [16]. These findings clearly demonstrate how detailed studies of the inhibitory action of FFA on virulence gene expression can elaborate new knowledge on how to develop antivirulence strategies against bacterial pathogens.

\section{Conclusion}

Naturally occurring medium- and long-chain FFAs represent an interesting and very useful group of antibacterial and antivirulence agents. In nature, they may act as signaling molecules to prevent activation of virulence gene expression under conditions where their induction is not required, such as in food. Studies on how these FFAs affect the growth and virulence of Listeria and other bacterial pathogens will most likely provide new ideas on how to combat bacterial pathogenic disease. The most encouraging potential clearly lies in the ability of subinhibitory 
concentrations of specific FFAs to inhibit virulence gene expression in important bacterial pathogens. Structural and functional studies on the inhibitory action of such FFAs on virulence will serve as inspiration for the future development of more efficient antivirulence agents. Most importantly, compounds acting by targeting virulence mechanisms represent a promising alternative to traditional antibiotics [8,9]. In contrast to antibiotics, agents acting by an antivirulence mechanism could reduce the pressure for the development of resistance by simply disarming the bacteria of its virulence factors while not preventing bacterial growth [8,9].

\section{Financial \& competing interests disclosure}

The laboratory of BH Kallipolitis is supported by grants from the VILLUM FONDEN and the European Union's Horizon 2020 research and innovation program under the Marie Sklodowska-Curie (List_MAPS; grant agreement number 641984). The author has no other relevant affiliations or financial involvement with any organization or entity with a financial interest in or financial conflict with the subject matter or materials discussed in the manuscript apart from those disclosed.

No writing assistance was utilized in the production of this manuscript.

\section{Open access}

This work is licensed under the Attribution-NonCommercial-NoDerivatives 4.0 Unported License. To view a copy of this license, visit http://creativecommons.org/licenses/by-nc-nd/4.0/.

\section{References}

1. Freitag NE, Port GC, Miner MD. Listeria monocytogenes - from saprophyte to intracellular pathogen. Nat. Rev. Microbiol. 7(9), 623-628 (2009).

2. Good JAD, Andersson C, Hansen S et al. Attenuating Listeria monocytogenes virulence by targeting the regulatory protein PrfA. Cell Chem. Biol. 23(3), 404-414 (2016).

3. Krajewski SS, Isoz I, Johansson J. Antibacterial and antivirulence effect of 6-N-hydroxylaminopurine in Listeria monocytogenes. Nucleic Acids Res. 45(4), 1914-1924 (2017).

4. Sternkopf Lillebæk EM, Lambert Nielsen S, Scheel Thomasen R, Færgeman NJ, Kallipolitis BH. Antimicrobial medium- and long-chain free fatty acids prevent PrfA-dependent activation of virulence genes in Listeria monocytogenes. Res. Microbiol. 168(6), 547-557 (2017).

5. Scortti M, Monzó HJ, Lacharme-Lora L, Lewis DA, Vazquez-Boland JA. The PrfA virulence regulon. Microbes Infect. 9(10), 1196-1207 (2007).

6. Reniere ML, Whiteley AT, Hamilton KL et al. Glutathione activates virulence gene expression of an intracellular pathogen. Nature 517(7533), 170-173 (2015).

7. Hall M, Grundström C, Begum A et al. Structural basis for glutathione-mediated activation of the virulence regulator protein PrfA in Listeria. Proc. Natl Acad. Sci. USA 113(51), 14733-14738 (2016).

8. Dickey SW, Cheung GYC, Otto M. Different drugs for bad bugs: antivirulence strategies in the age of antibiotic resistance. Nat. Rev. Drug Discov. 16(7), 457-471 (2017).

9. Johnson BK, Abramovitch RB. Small molecules that sabotage bacterial virulence. Trends Pharmacol. Sci. 38(4), 339-362 (2017).

10. Petrone G, Conte MP, Longhi $\mathrm{C}$ et al. Natural milk fatty acids affect survival and invasiveness of Listeria monocytogenes. Lett. Appl. Microbiol. 27(6), 362-368 (1998).

11. Chatterjee A, Dutta PK, Chowdhury R. Effect of fatty acids and cholesterol present in bile on expression of virulence factors and motility of Vibrio cholerae. Infect. Immun. 75(4), 1946-1953 (2007).

12. Lowden MJ, Skorupski K, Pellegrini M, Chiorazzo MG, Taylor RK, Kull FJ. Structure of Vibrio cholerae ToxT reveals a mechanism for fatty acid regulation of virulence genes. Proc. Natl Acad. Sci. USA 107(7), 2860-2865 (2010).

13. Plecha SC, Withey JH. Mechanism for inhibition of Vibrio cholerae ToxT activity by the unsaturated fatty acid components of bile. J. Bacteriol. 197(10), 1716-1725 (2015).

14. Childers BM, Cao X, Weber GG, Demeler B, Hart PJ, Klose KE. N-terminal residues of the Vibrio cholerae virulence regulatory protein ToxT involved in dimerization and modulation by fatty acids. J. Biol. Chem. 286(32), 28644-28655 (2011).

15. Golubeva YA, Ellermeier JR, Cott Chubiz JE, Slauch JM. Intestinal long-chain fatty acids act as a direct signal to modulate expression of the Salmonella pathogenicity island 1 type III secretion system. MBio 7(1), e02170-15 (2016).

16. Woodbrey AK, Onyango EO, Pellegrini M et al. A new class of inhibitors of the AraC family virulence regulator Vibrio cholerae ToxT. Sci. Rep. 7, 45011 (2017). 
